\title{
Narrative der Integration und Assimilation im Film ${ }^{1}$
}

ÖZKAN EZLI

\section{ZUSAMMENFASSUNG}

Wir schreiben das Jahr 1987 und der Hauptdarsteller Jan (Uwe Bohm) hat sich in Hark Bohms bekanntem Film Yasemin in die Hauptdarstellerin, in die 17-jährige Deutsch-Türkin Yasemin (Ayşe Romey) verliebt. Er wird irgendwann gegen Ende des Films Türkisch lernen, um ihr näherzukommen, um sie besser zu verstehen. Jedoch weiß bis dahin jeder aufmerksame Filmzuschauer, dass dieses Vorhaben nicht wirklich nötig ist, denn Yasemin spricht im Film kaum Türkisch, dafür aber ein akzentfreies perfektes Deutsch, trägt kein Kopftuch, ist im Judoverein aktiv, will Kinderärztin werden und verhält sich, diese Attribute zusammengenommen, selbstbewusst und modern. Man könnte sie kognitiv und strukturell als assimiliert bezeichnen. Warum Jan unter diesen Umständen Türkisch lernt, ist entweder als eine rein symbolische Geste gegenüber Yasemin zu verstehen oder aber sie hängt mit einer Form der Kulturalisierung und Folklorisierung zusammen, die weit über die Bedürfnisstruktur der Beziehung, aber auch über die der dargestellten türkischen Familie in Yasemin hinausgeht.

1 Siehe grundlegend zum Ansatz, gesellschaftspolitische Debatten und soziologische Modelle und Konzepte in ihren Prägungen und Wirkmächtigkeiten als Narrative zu lesen: Koschorke, Albrecht: Wahrheit und Erfindung. Grundzüge einer allgemeinen Erzähltheorie. Frankfurt a. M.: Fischer 2012. 
Knapp zwanzig Jahre später verliebt sich der Hauptdarsteller Götz (Florian David Fritz) in der preisgekrönten Multi-Kulti-Komödie Meine verrückte türkische Hochzeit in die Deutsch-Türkin Aylin (Mandala Tayde). Ebenso wird er einen Zugang zur anderen Kultur finden, jedoch ist es diesmal nicht die Sprache, sondern wir sehen Götz nach knapp der Hälfte des Films einen Spiegelartikel zu Osama bin Laden lesen. Grund hierfür ist, dass Götz nach Auffassung von Aylins Familie zum Islam konvertieren muss, will er sie heiraten. Auch wenn wie im Film Yasemin die weibliche Hauptdarstellerin weder als eine traditionell-türkische Frau, noch als eine überzeugte Muslima gezeigt wird, kann das kulturelle Bemühen von Götz nicht einfach als eine symbolische Geste interpretiert werden. Vielmehr folgt es komödiantisch-parodistisch der aktuellen bundesrepublikanischen Politik des Forderns und Förderns, die zum einen Assimilation und Kultur an gesetzgeberischen Praktiken festmacht, zum anderen den Islam als zentralen anzuerkennenden Bestandteil der Integration begreift (Deutsche Islam Konferenz).

Zwei Dekaden trennen diese beiden Filme voneinander und sie zeigen differente Narrative der Assimilation, Integration und Diversität auf, die aus höchst unterschiedlichen deutsch-deutschen und deutsch-türkischen Konstellationen hervorgegangen sind. Zwischen dem klassischen Integrationsnarrativ, das auf eine ziel- und prozessorientierte Form der Assimilation setzte und der aktuellen kulturalisierenden Erzählung vom angekommenen deutschen Muslim (Schäuble 2009), gibt es ein dazu querstehendes und vielschichtiges Feld von Prozessen, die ich mit der vergleichenden Analyse der Filme Yasemin und Meine verrückte türkische Hochzeit mit ihren jeweiligen gesellschaftspolitischen Rahmungen skizzieren möchte. Im Vordergrund der Analyse wird die Beobachtung stehen, welche ähnlichen und unterschiedlichen Nah- und Distanzbeziehungen die Akteure zu kulturellen Kennzeichen in den 1980er und heute unterhalten und wie kohärent oder inkohärent diese Verhältnisse in Narration und Rezeption sind (vgl. Ezli 2012a: 9-13; Koschorke 2012: 38-44). Darauf aufbauend werde ich der Frage nachgehen, wohinein die Akteure sich eigentlich integrieren wollen und sollen? Welches Feld und welche Form der möglichen oder unmöglichen Ankunft wird in den filmischen Narrativen konstituiert, die mit den jeweiligen politischen Integrationsnarrativen korrelieren und auch über sie hinausweisen. 


\section{EINLEITUNG}

Die muslimischen Einwanderer ,können nicht gegen ihre Religion, sondern nur mit dieser in eine westliche Gesellschaft integriert werden“, resümiert Jürgen Habermas in seinen Überlegungen zur Dialektik der Säkularisierung (Habermas 2008: 41). Mit dem Fokus auf aktuelle europäische Integrationspolitiken hält er auch vor, dass es einen bestimmten Denk- und staatlichen Konstitutionsfehler in der langen Geschichte der europäischen Säkularisierung und Modernisierung gab, nämlich Religion als Partizipations- und Sinnstifter aus dem öffentlich-politischen Raum ausgeschlossen zu haben.

Den Anfang nahm diese Trennung mit den Konfliktschlichtungen nach den europäischen Konfessionskriegen im 16. und 17. Jahrhundert, in denen die Staatsgewalt „zu weltanschaulich neutralem“ Handeln genötigt war. „Sie musste die streitenden Parteien entwaffnen, Arrangements für ein friedlich-schiedliches Zusammenleben der verfeindeten Konfessionen erfinden und deren prekäres Nebeneinander überwachen. In der Gesellschaft konnten sich die gegnerischen Subkulturen dann so einnisten, dass sie füreinander Fremde blieben." (Habermas 2008: 39) Dieser Modus Vivendi überdauerte auch die grundlegend säkularistische Neuordnung des Staates in Europa im späten 18. Jahrhundert und hat mitunter dazu geführt, dass die katholische Kirche sich ,bekanntlich erst mit dem zweiten Vaticanum im Jahre 1965 zu Liberalismus und Demokratie bekannt hat" (Habermas 2008: 44). ${ }^{2}$

Diese Ordnung des Nebeneinander und des „einnisten lassens“ zeige sich aber auch im ,falsch verstandenen“ Multikulturalismus der 1980er und 1990er Jahre, in dem man von einer , >>Inkommensurabilität<< von Weltbildern, Diskursen oder Begriffsschemata“ ausging (Habermas 2008: 42). Eine gelingende Integrationspolitik braucht jedoch nach Habermas ein anderes Verhältnis von demokratischem Staat, Zivilgesellschaft und subkultureller Eigenständigkeit. Dafür müssen die „Kulturen ihre individuellen Mitglieder aus der Umklammerung entlassen, damit diese sich in der Zivilgesellschaft gegenseitig als [...] Träger und Mitglieder desselben politischen Gemeinwesens anerkennen können“ (Habermas 2008: 39). So geht es hier

2 Ein Prozess, den der Islam lokal, national und global noch vor sich hat und nach Habermas in den aktuellen Integrationspolitiken europaweit eine zentrale Rolle spielt, wenn Integration gelingen soll. (Habermas 2008: 44). 
um eine neue Bindung von Staats- und Gesellschaftsbürger, mit der alteingesessenen Deutschen und muslimischen Zuwanderern Interpretationen des Verhältnisses von Glauben und Wissen jeweils aus ihrer Sicht eingeräumt werden, „die ihnen ein selbstreflexiv aufgeklärtes Miteinander möglich macht" (Habermas 2008: 46). Habermas Hoffnung hier ist, durch eine Expansion der Selbstreflexion, Strukturen von Parallelgesellschaften aufzubrechen; jedoch eine Selbstreflexion, die nach ihm in einer postsäkularen Zeit ohne Religion nicht mehr zu denken ist.

Sein Credo der Partizipation selbstreflexiver religiöser Akteure fügt sich nahtlos in die Bahn einer Integrationspolitik, die seit 2005 mit den Integrationsgipfeln, den Islamkonferenzen ab 2006 (Tezcan 2012) und dem Nationalen Integrationsplan aus dem Jahre 2008 die deutsche politische Agenda bestimmt. Und ähnlich wie Habermas, hielt der damalige Innenminister Wolfgang Schäuble ein Jahr nach der Veröffentlichung von Habermas Säkularisierungsaufsatz narrativ fest, dass die türkischen Gastarbeiter, die vor knapp fünfzig Jahren aus der Türkei kamen, nach vielen Verwirrungen und Sprachlosigkeiten zwischen Migranten der ersten und zweiten Generation und dem Einwanderungsland, nun in der Mitte der deutschen Gesellschaft als deutsche Muslime angekommen seien (Schäuble 2009). Die Ankunft, die der ehemalige Innenminister hier anspricht, kann jedoch nach einer 50jährigen Migrationsgeschichte keine materiell-körperliche mehr sein, sondern ,nur“ noch eine identitätspolitisch-symbolische.

Die viel beschworenen Zwischenräume deutsch-türkischer Bindungen und ihre Problematisierungen in den 1980ern und 1990ern in Politik und Medien, Pädagogik, Sozial- und Geisteswissenschaften, in Literatur und Film, scheinen bei dieser langen Odyssee nach Schäuble und Habermas überwunden. An die Stelle des türkischen Gastarbeiters der ersten Generation, der in dem Poem Was will Niyazi in der Naunynstraße? (1973) von Aras Ören noch den Wunsch äußerte, Amerikaner werden zu wollen (Vgl. Ören 1973: 28), und an die Stelle der Kanaken der 1990er, die systemkritisch mit einer Mischung aus Dichtung und Wahrheit eine sprachpolitische globale Identität jenseits nationaler oder kultureller Zuschreibung evozierten (Vgl. Y1ldız 2009: 195), ist ein neues Subjekt getreten und gesetzt worden: das muslimische Kultursubjekt, das sein Heil scheinbar nicht mehr im materiell besseren Leben oder im Protest findet, sondern in einer angeblich ursprünglichen Identität als Muslim (Vgl. Ezli 2012a: 10 f., 183 f.). 
Politische Ereignisse wie Integrationsgipfel und Islamkonferenzen erzählen eine Entwicklungsgeschichte, die alle Ereignisse, die sich zwischen dem Anfang als Bruch und Misskommunikation und der Ankunft in der Mitte der Gesellschaft ereignet haben und ereignen, für „Verkleidungen [und] für bloße Zufälle [hält]; sie möchte alle Masken abtun, um endlich eine erste Identität aufzudecken“ (Foucault 2009: 85), die des Einwanderers, der ohne seine Religion nicht zu integrieren ist. Dabei finden wir in den klassischen Migrations- und Integrationstheorien eine gegenteilige Orientierung vor. Die Geschichte des Einwanderers ist danach vielmehr die der Assimilierung und nicht der Entdeckung der „ersten Identität“. Denn nach klassischer Lesart folgt auf die kognitive, strukturelle und soziale Assimilation die identifikative, mit der der ursprüngliche Migrant oder spätestens die dritte Generation sich mit der Kultur der Mehrheits- und nicht mit der Religion aus der Herkunftsgesellschaft identifiziert (Park 1928, Gordon, 1963; Esser 1980 und Han 2009: 36-46). In der Öffentlichkeit und Teilen der Sozialwissenschaften wird demgegenüber seit den 1990er Jahren Integration auch als wechselseitiger Prozess verstanden, im Zuge dessen sich auch die aufnehmende Gesellschaft anzupassen und zu verändern habe eine Sichtweise die vehement auch von migrationspolitischen deutschtürkischen Dachverbände vertreten wurde (Rauer 2008). ${ }^{3}$ Integration ist und soll hier keine Assimilation sein, die die Aufgabe der Herkunftskultur impliziert. Es soll weder um kulturelle Homogenität, noch um die Ausschließung kultureller Prägungen gehen. Dennoch geht es wie beim Assimilationskonzept um eine ähnliche Bewegungsabfolge, die von einem kritisierten Ist-Zustand einer Gesellschaft zu einem erwünschten Soll-Zustand führen soll. ${ }^{4}$

Im anliegenden Vergleich wird die Analyse zeigen, dass die Erzählweisen der Integration und Assimilation in diesen Filmen über die jeweiligen gesellschaftspolitischen Rahmungen und Konzepte hinausweisen und eine kulturwissenschaftliche Reflexion über den Zusammenhang der Artikulationsformen von filmischen Erzählungen und politischer Kultur ermöglichen.

3 Wissenschaftlich fand diese zweite Sichtweise vor allem später Ausdruck in postkolonialen und transnationalen Ansätzen (Bhaba 1994; Faist 2000).

4 Siehe hierzu ausführlicher die Einleitung in diesem Band. 


\section{Ein Clash der NarRative oder die blockierte INTEGRATION}

Hark Bohms Yasemin war auf vielen Ebenen ein erfolgreicher Film. 1989 erhielt er den renommierten Bundesfilmpreis in Gold, im gleichen Jahr erhielten die Hauptdarsteller je den Bayerischen Filmpreis als beste Nachwuchsdarsteller, beim Deutschen Jugend-Video-Preis bekam der Film den 2. Preis und 1991 folgte der deutsche Fernsehpreis (vgl. Matthias 2002: 35). Zugleich war Yasemin ein Film, der von Anfang der 1990er bis Anfang des neuen Jahrtausends in den Curricula der Mittel- und Oberstufe in Deutschland oft zu finden war und so als beliebtes Lehrmaterial eingesetzt wurde.

Als pädagogisch „,besonders wertvoll“ wurde an dem Film geschätzt, dass er alltagsnah das Leben der türkischen Einwanderer zeige und zugleich durch seine differenzierte Darstellung dieser Lebenswelt eine multiperspektivische Sicht auf ein bis dahin klischeebeladenes Thema ermögliche (vgl. Ehlers 1993 und Matthias 2002). Er war ein Lieblingsfilm der Pädagogen, die in Yasemin nicht den „Konflikt zwischen Liebe und Zwängen der Tradition (Familien-Ehre)“ als den eigentlichen sahen, sondern ,Zwischen diesen und der Freiheit zur persönlichen Entwicklung“ (Matthias 2002: 36). So realisiere dieser Film Empathien auf kultureller und auf individueller Ebene zugleich (ebd.: 37). Bei einer Befragung von Schülern, wie dieser Film auf sie gewirkt habe, heißt es Anfang der 1990er, dass diese ,sowohl emotional berührende Szenen wie auch abstrakte Themen“ angeben (Ehlers 1993: 145). Interessanterweise ging der Regisseur Hark Bohm selbst während der Postproduktionsphase seines Films ähnlich vor. Er zeigte ihn Schülern und Studenten und berücksichtigte ihre Kritik und ihren Zuspruch beim finalen Schnitt des Films (Bohm 1988: 2).

So pädagogisch das alles klingen mag, betonte Bohm auch, dass er mit Yasemin keinen belehrenden Film gedreht, vielmehr eine archaische Geschichte erzählt habe (Siehe Bohm 1988: 4), in der ein Vater durch die Angst um seine Tochter anfängt, sie vor Fremden zu schützen. Nicht kulturell, sondern existentiell orientiert, beinhaltet Bohms ästhetisch-politisches Credo auch keine explizit inter- oder multikulturelle Bestimmung. Denn der Künstler hat für ihn ,in seiner archaischen Funktion [...] die Aufgabe, eine Gemeinde zu stiften, und in der Versammlung der Gemeinde wirst du aus deiner Angst, isoliert zu sein, nicht dazuzugehören, erlöst“ (aus: Visarius 1995: 124). Nicht eine zu erreichende Gesellschaft, wie sie der klassische 
Begriff der Integration verlangt, steht hier im Vordergrund, vielmehr eine zu bildende Gemeinschaft. Nicht das geregelte Auskommen miteinander, sondern ein intimes Verstehen des Anderen.

Warum dieser Film dennoch als ein Beitrag des interkulturellen Verstehens für die deutsche Gesellschaft gelesen wurde, hat zum einen mit den Inkohärenzen des Films, seiner Dramaturgie, und zum anderen mit Interpretationen zu tun, die auf der Verwechslung der sozialen Entitäten von Gemeinschaft und Gesellschaft beruhen. Diese Inkohärenzen füllen die Leerstellen des Films zwischen Emotion und Abstraktion, zwischen Akteuren und Kulturen und generieren Narrative der Integration, die sich strukturell in wissenschaftlichen und gesellschaftspolitischen Debatten der 1980er wiederfinden.

$\mathrm{Zu}$ Anfang des Films wird der deutsch-deutsche Protagonist Jan als neues Mitglied in einem Judoverein vorgestellt, in dem auch Yasemin trainiert und Mitglied ist. Um seinen neuen Freunden zu imponieren, schließt Jan nach dem Training mit ihnen eine Wette ab, dass es keine Braut gebe, die er innerhalb von zwei Tagen nicht auf der Matte habe. Seine zufällige Wahl, er dreht sich, die Augen geschlossen, Arm und Zeigefinger ausgestreckt, fällt auf Yasemin. Nachdem seine Sportkollegen ihm sagen, dass Yasemin härter sei als Granit und er von ihr nicht mehr als ein „Guten Tag“ erwarten könne, geht Jan nochmal nach draußen und zielt mit dem Finger symbolisch als Pistole auf den mit Yasemin und ihrem Vetter vorbeifahrenden blauen Ford Transit, dass er sie schon kriegen werde. Diese Form des penetranten Auftretens wird Jan erst ablegen, nachdem er sich nach eigener Aussage in Yasemin verliebt hat.

Nach dieser Einstiegssequenz wird Yasemin auf dem Markt und danach in der Schule als eine selbstbewusst und souverän agierende Person gezeigt, die jedoch mit gewissen Einschränkungen auskommen muss. Ihre Lehrerin fragt sie, ob ihr Vater eingewilligt habe, was ihren Wunsch zu studieren betrifft, da sie noch nicht volljährig sei. Nach dem Schulunterricht auf dem Nachhauseweg zieht sie ihr Kleid nach unten, dass man ihre Beine nicht sieht. Ihr Vetter wird sie gleich abholen, der sie auch auf dem Markt begleitet hatte. Jedoch wartet auch noch ein anderer Mann auf sie, Jan, der ihr mit seinem Motorrad nachgeht und sie fragt, wann sie den zusammen mal ausgehen könnten. Yasemin ist von seiner Anfrage angetan, versteckt sich jedoch bei der nächsten Gelegenheit in einem Hauseingang vor ihm. Noch nicht klar, ob aus Koketterie oder nicht, erhärtet sich der Verdacht, dass 
dieses Verstecken mit ihrem Vetter zusammenhängt, der dann auftaucht und sie sucht.

Dieses gleich zu Anfang angelegte Spannungsfeld, zwischen Familie, an diese gebundenen (Yasemin) und ungebundenen (Jan) Individuen, wird ab der Mitte des Films expandieren, wenn bei der Hochzeitnacht von Yasemins älterer Schwester Emine (Nursel Köse) der Beweis ihrer Jungfräulichkeit ausbleiben wird. Bis zu diesem Ereignis ist das hier kurz skizzierte Spannungsfeld zwar sichtbar, jedoch bleibt es auch verhandelbar. Denn weder der Vater noch der Vetter werden in der ersten Hälfte des Films als Tyrannen dargestellt. Im Gegenteil zeichnen sie sich in der Darstellung von Bohm durch eine besondere Fürsorge für Yasemin aus. Beispielsweise wird Yasemins strenger und konservativer Onkel, der bei niemandem in der Familie beliebt ist, immer wieder von seinem Bruder Yusuf spielerisch beschwichtigt, wenn dieser Yasemins Judotätigkeit oder ihren Wunsch zu studieren, dem ihr Vater öffentlich auf der Hochzeit nachgibt, als unsittlich für eine Frau kritisiert. Judo lerne sie für Ihre Selbstverteidigung und seine Tochter wolle Kinderärztin werden, was soll daran falsch sein, erwidert Yusuf seinem Bruder. Jedoch verändert sich dieser herzliche verhandlungsoffene Raum zwischen Tochter und Vater grundlegend, als der Nachweis von Emines Jungfräulichkeit ausbleibt.

In seiner Ehre getroffen, geht es dem Vater anschließend darum, den Rest seiner Familie vor einem weiteren Eindringen des Außen zu schützen, das sich von der Lehrerin Yasemins über den Ort St. Pauli bis hin zu Jan erstreckt. Dieses neue Verhältnis zwischen Innen und Außen führt nun durch seine harte Grenzziehung zwischen der türkischen und deutschen Welt, das zuvor porös, heterogen und handlungsoffen war, am Ende dazu, dass Yasemins Vater seine Tochter in die Türkei zurückbringen will, um sie zu schützen. Sein Versprechen, dass sie Kinderärztin werden darf und eine Zukunft in Deutschland haben kann, kehrt sich ins Gegenteil. In der Schlusssequenz des Films bringen Vater und Neffe mit dem blauen Ford Transit Yasemin an einen unbestimmten Ort in Deutschland. Es ist Nacht, wir sehen türkische Männer tanzend um eine Feuerstelle. Männer, die sie in die Türkei zurückbringen sollen. Doch Jan ist dem blauen Ford Transit mit seinem Motorrad gefolgt, Yasemin kann sich mit einem Messer in der Hand von den Männern entfernen und mit Jan fliehen. Mit ersichtlicher Trauer, ihre Familie verlassen zu müssen, sehen wir Yasemin in der letzten Einstel- 
lung im Film in dunkler Nacht auf dem Rücksitz des fahrenden Motorrads, Jan umarmend. Ein offenes, jedoch kein zukunftsversprechendes Ende.

Der Prozess und narrative Verlauf, der nach dem Fehlen des Brautblutes einsetzt und den Film in zwei unterschiedliche soziale Ordnungen trennt, beschreibt der Regisseur Hark Bohm selbst als eine nicht nachvollziehbare Abfolge und setzt dabei Jans Perspektive mit der der Mehrheitsgesellschaft gleich.

„Yasemin fühlt sich als Deutsche, denkt und handelt wie eine moderne, intelligente westeuropäische Frau. (...) Erst durch die Liebesgeschichte mit Jan erfährt Yasemin wirklich, was es heißt, eine türkische Tochter zu sein. Erst dann zerbricht das fragile Gleichgewicht zwischen türkischer Tochter und westdeutscher Gymnasiastin und wird zu einem offenbar unlösbaren Konflikt. Und wir müssen, wie Jan, staunend und hilflos mit ansehen, wie sich dieser Konflikt fast gesetzmäßig verschärft. “5

Woher diese Gesetze kommen, die ein Regelwerk in diesen Film einbrechen lassen, wird für uns auch Jan herausfinden. Auf seiner Suche in Hamburg nach einem Deutsch-Türkischen Wörterbuch findet er zunächst kein Buch zur türkischen Grammatik und Vokabeln, sondern einen Bildband zur Türkei. Das erste Bildmotiv, das wir sehen, ist die Blaue Moschee in Istanbul mit dem Wasser des Goldenen Horns im Hintergrund. Jan blättert weiter und das Bild, das auf die Moschee folgt, ist ein fotografisches Stillleben türkischen Dorflebens. Wir sehen im Vordergrund mehrere Frauen bei der Arbeit mit dem Rücken zur Kamera in traditioneller Dorfkleidung, in der Mitte links ein paar Männer auch bei der Arbeit und im Hintergrund Häuser, die auf ein Dorf, höchstens auf eine Kleinstadt schließen lassen. Keine der gezeigten Personen blickt in die Kamera, es sind alle mit ihrer Arbeit beschäftigt und haben ihren Ort und ihre Funktion. Sie sind eingebunden in ein Kollektiv und stellen eine bestimmte Sozialstruktur dar.

Die Türkei ist in diesem Film ein Dorf, und auf diese Relation wird im zweiten Teil des Films immer stärker Bezug genommen. Zum ersten Mal durch die Drohung des Vaters als Reaktion auf die Ehrverletzung, die ganze Familie in die Türkei zurückzubringen, falls sich Frau, Töchter und Vetter nicht mehr ehrenhaft verhalten sollten. Eine Drohung, die niemanden aus der Familie erfreut. Als sich Jan und Jasemin danach heimlich auf einem Dampfer treffen, antwortet sie auf seine Frage, warum sie sich denn hier

5 Zitat aus: DVD Hark Bohm, Yasemin, Zweitausendeins Edition 3/1988. 
treffen würden, weil sie ,das zufällige Produkt einer Kopulation zweier anatolischer Dorfmenschen“ sei (Bohm 1988). Später wird dann der Judolehrer Dursun zurechtweisen, als dieser im Training Jan fast erwürgt, dass er hier nicht in der Türkei sei. Für Yasemin, die das mitbekommt, eine verletzende Aussage, die auch ihr Verhältnis zu Jan beeinflussen wird.

Der Konnex von türkischer Gastarbeitermigration nach Deutschland und türkischem Dorf spielt in den zeitgleich entstandenen ethnologischen Arbeiten von Werner Schiffauer eine zentrale Rolle. Diese Verbindung ist nach ihm die Grundlage des Clash-of-Culture, der die türkische Gastarbeitergeneration der ersten und zweiten Generation ausgesetzt war (Schiffauer 1979: 103). Für den Ethnologen der 1980er Jahre vor allem ein Konflikt der die Innen/Außen-Unterscheidung, die auf dem Dorf für viele Migranten der ersten Generation üblich war, in sein Gegenteil verkehrt. Im Dorf ist die Familie eine Produktions- und Konsumtionseinheit, die auf den inneren $\mathrm{Zu}$ sammenhalt stark angewiesen ist. Für jeden Akteur ist sie die Hauptreferenz und die politische Entität im Dorf, die es aus Eigen- und sozialem Interesse zu schützen gilt. Die Struktur in der Familie im Dorf ist hierarchisch und von klaren Verhaltensregeln, besonders gegenüber dem männlichen Familienoberhaupt, geprägt. Nach außen hin ist es wichtig, dass diese Struktur der Achtung beibehalten wird. Ein wichtiger Gradmesser hierfür ist die Unversehrtheit der Ehre (Namus). ${ }^{6}$ Denn im Herkunftsort Dorf weist besonders die Funktion und der Begriff der Ehre jedem Mann ,einen Bereich persönlicher Integrität und Würde [zu], der die eigene körperliche

6 Die Funktion und die Reichweite des Begriffs Ehre ist im türkischen Kontext vielfältig. Dabei handelt es sich um drei Begriffe, die semantisch ineinander greifen und das soziale Wirkungsfeld von Namus markieren. Der Begriff selbst bezeichnet einen Zustand, der nicht erworben werden kann, sondern nur verloren. Die Ehrhaftigkeit und Reinheit der Frauen in der Familie ist damit verbunden. Der Verlust der Ehre bedeutet nach Innen wie nach Außen den Verlust von sozialem Status. Sayg (Achtung, Ehre, Respekt) ist respektierendes und angemessenes Verhalten, das man älteren Personen gegenüber erbringt. Sie muss immer wieder bestätigt werden, ist also eine aktive Handlung. Der letzte wichtige Begriff in diesem Zusammenhang ist Şeref (Ehre, Wert, Würde), das durch positive Eigenschaften wie „Großzügigkeit, Wissen, Reichtum, Macht und Einfluss" erworben werden kann. Sie stellt einen Wert dar. (Siehe Alabay 2012: 96- 
Unversehrtheit wie die der Familienangehörigen umfasst" (Schiffauer 1979: 70). So ist ein ,ehrenhafter“ Mann jemand, ,der seine Frau zu verteidigen vermag, der Stärke und Selbstbewusstsein zeigt, politische, die äußere Sicherheit seiner Familie garantierende Fähigkeiten“" (Schiffauer 1979: 74). Dieser aktiven Rolle gegenüber steht eine eher passive der Frau, die „ehrenhaft" ist, wenn sie keusch bleibt (Schiffauer 1979: 72; siehe auch Alabay 2012: 98). Jede Form des sexuellen Austauschs außerhalb der Ehe verletzt nicht nur die Ehre der Frau, sondern auch die der männlichen Mitglieder der Familie (Schiffauer 1979: ebd.; siehe auch Alabay 2012: 96 f.). Das Selbstverständnis des Mannes ist nach Außen von der Unterscheidung stark-schwach bestimmt und die der Frau von der Dichotomie rein-unrein (Schiffauer 1979: 75).

Die Achtung, die jede Familie einer anderen gegenüber erbringt, erfolgt nach den Verhaltenscodes reziprok und garantiert eine friedliche und solidarische Nachbarschaft. Wenn jedoch die Grenze der Ehre und Achtung verletzt wird und es zu Konflikten kommt, werden diese entweder über die staatlichen Gerichte geschlichtet oder aber, was häufig der Fall ist, nimmt nicht selten die gemeinsame Religion, der Islam, eine schlichtende und verbindliche Funktion in den Konfliktfällen ein. „Die Klage, dass vom Islam nichts mehr $>>$ geblieben sei $<<$ (Islamiyet kalmadı burada), wird im Dorf immer dann laut, wenn das Gemeinwesen in einzelne, nur auf die eigene Ehre bedachte Gruppen zu zerfallen droht“ (Schiffauer 1979: 68). Dieses Innen (Familie) und Außen (andere Familien), gerahmt von der Sozialstruktur des türkischen Dorfs, ist in der Fremde nicht mehr eine haltbare Leitunterscheidung, denn die Migration nach Deutschland bringt für die Familie zwei Veränderungen mit sich: „Die Familie ist nicht mehr Produktions- und Konsumtionseinheit, die anderen, gleichfalls unabhängigen Familien in Gegenseitigkeitsverhältnissen gegenübersteht, sie ist jetzt eingebettet in komplexe und funktionale Zusammenhänge.“ Innen und Außen stehen nicht mehr in Korrelation zueinander, sind voneinander getrennt und das Familienoberhaupt fühlt sich nur noch der Familie verpflichtet (Schiffauer 1979: 102, 104).

Als Yasemin nach der nicht erfolgreichen Brautwache zur Hochzeit ihrer Schwester erst einen Tag später nach Hause kommt, weil Sie dem Stress zu Hause ausweichen wollte, ist der bis dahin herzlich dargestellte Vater ein anderer und nimmt auch das Versprechen, dass Yasemin studieren könne, zurück mit der Begründung, dass er gestern noch ein Mann mit Achtung 
(Şeref) und Ehre (Namus) gewesen und dies jetzt nicht mehr der Fall sei. Da die Frau die Ehre der Familie repräsentiert, gilt es jetzt, Yasemin durch Beobachtung und Kontrolle nach Auffassung des Vaters zu schützen. Dursun soll nun überall, wohin Yasemin geht, dabei sein. Der Raum der Verhandlungen ist nun eingeengt, denn das Außen ist in die Familie eingedrungen und diese ist nun zu verteidigen, um die Ehre nicht gänzlich zu verlieren. Den Kontakt zur älteren Tochter bricht der Vater ab und zwingt auch die anderen Familienangehörigen dazu.

Dieser Verhaltenswandel des Vaters von einer verständigen und herzlichen Person zu einem Tyrannen wird für den Zuschauer erst dann kohärent, wenn wir den Diskurs um Dorf und Ehre in die Interpretation zwischenschalten. Denn das, was im Nachhinein mit Jans Suche nach einem Wörterbuch in die Familie gelegt wird, dass sie anderen sozialen Ordnungen folgt als die westlich-aufgeklärte Gesellschaft, zeigt sich in der ersten Hälfte des Films nur luzide. Und auch die dargestellten deutsch-türkischen Kontakte sind zunächst keineswegs konfrontativ. Beispielsweise als Yasemin sich auch aus Koketterie heraus vor Jan versteckt und ihr Vetter sie sucht, begegnen sich Jan und Dursun und beide verheimlichen auf eine recht schüchterne Art, wen oder was sie suchen. Sie fangen an, über Jans Motorrad zu reden. Auf der Hochzeit von Yasemins Schwester begegnen wir einer vielfältigen Lebensfreude, die mit einer besonders bunten Farbdramaturgie akzentuiert wird. Wenige Frauen tragen Kopftuch und die, die eines aufhaben, tragen es traditionell lose am Kopf. Hier findet sich kein Akteur, ob Mann oder Frau, dessen Freiheit beschnitten wird, weil ein Ehrenkodex einzuhalten wäre. Im Gegenteil steht die Hochzeitssequenz in diesem Film für eine äußerst integrative Kontaktzone, die am Ende nur einen Ausgeschlossenen kennt. Denn Jan ist im Unterschied zu den nahen Verwandten, näheren Bekannten und Yasemins männlichen und weiblichen Freunden aus dem Judoverein nicht eingeladen. Er kommt aber trotzdem und wird auf Yasemins Wunsch hin aus der Hochzeitgesellschaft ausgewiesen. Auch die Brautwache bietet Möglichkeiten den Ehrenkodex zu umgehen. Wir sehen in dieser Filmsequenz zwei ältere türkische Damen mit Yasemin rauchend zusammensitzen. Die Damen erzählen sich selbst und Yasemin eine Geschichte wie ein junger Mann geschickt den Blutbeweis erbringt, obwohl seine Frischvermählte nicht mehr jungfräulich ist. Wie Alexander der GroBe schneidet er sich in den Finger und befleckt damit das Bettlaken und zeigt diesen Beweis der Jungfräulichkeit den draußen Wartenden. Von die- 
ser Geschichte sind die zuhörenden Frauen angetan und empfinden sie aber auch als eine normale Strategie der kulturellen Verhandlung. Man sollte sie nur nicht öffentlich machen. Eine Verhandlung, die Yasemins Schwager nicht beherrscht, denn er tritt aus dem Schlafzimmer und hat nichts in der Hand. Später werden wir erfahren, dass es nicht an Yasemins Schwester lag, sie war und ist Jungfrau, sondern an der Impotenz des Schwagers, der dies aus Ehre nicht bekennen kann.

Dieser Bruch zwischen der Geschichte der Umgehung und der Verhandlung kultureller Gesetze und der Wirklichkeiten im Film sowie der Unfähigkeit des Schwagers strategisch zu lügen, bringt ein neues Verhältnis von Kultur und Akteur in den Film, das die Befolgung kultureller Gesetze verlangt und die Akteure dadurch ihre soziale Fluidität verlieren lässt. Und warum diese Fluidität sich identitätspolitisch in der Figur des Vaters verkrustet und klare kulturelle Grenzziehungen einsetzen, liegt nicht allein am Ehrenkodex, sondern auch an einem Clash der Narrative, an einem Mangel, wo hinein die Möglichkeiten des kulturellen Verhandelns sich bewegen sollen. Die Ehre und das Dorf als geschlossene Einheit sind die eine Seite, sie werden aber zu einem inkohärenten Problem, wenn Menschen mit ihren vielschichtigen Bedürfnisstrukturen gezeigt werden und nicht klar ist, an welchem Ort der Ankunft sie diese stabilisieren können. Hier zeigt sich Jan als emblematische Gegenfigur. Denn wenn auf der türkischen Seite detailliert ein familiärer Alltag gezeigt wird, Töchter gehen in die Schule, Vater arbeitet, Mutter kocht für die Kinder, Töchter helfen dem Vater im Geschäft, sie besuchen Ihren Judounterricht usw., ist ein Alltag in Jans Welt außer dem Judoverein nicht existent. Es gibt eine einzige kurze Sequenz, in der Jan sich zu Hause für die türkische Hochzeit anzieht und sein Vater ihn zum Abendessen erwartet. Der Vater bemerkt, dass Jan seinen Anzug angezogen hat und versucht ihm diesen abzunehmen. Jan flieht mit seinem Motorrad, der Vater hinterher, der ihn nicht mehr erwischt und beim zurückgehen ins Haus slapstickartig stolpert. Eine deutsche Vaterfigur, die mehr als nur eigenschaftslos wirkt. Ansonsten gibt es keine einzige Szene in diesem Film, die Jan, dem Deutschen, eine Zugehörigkeit oder einen stabilen Ort zuweist. Aber auch die anderen deutschen Protagonisten, die Lehrerin und die blonde Freundin Yasemins, sind in ihrem Auftritt und in ihrer Funktion nur mit der türkischen Familie beschäftigt. Sie haben in diesem Film auch kein Alltagsleben. Das Deutsche definiert sich hier nicht, unterliegt keiner 
Reflexion und zeigt auch keinen Raum, wo es beheimatet sein könnte. ${ }^{7}$ Und so stellt sich am Ende des Films auch nicht eine Erleichterung oder Freude ein, dass endlich die Liebenden zueinander gefunden haben, sondern vielmehr die Sorge, wohin dieses Paar eigentlich soll. Ähnlich drängt sich bei diesem Film auch die Frage auf, wohin das heterogene soziale Geflecht soll, das den ersten Teil des Films bestimmt, das von sozialen Verhandlungsräumen und vom ,guten“ und „warmherzigen“ Ausländer geprägt ist.

Im Unterschied zu Habermas“ und Schäubles klaren und Konsistenz generierenden Aussagen zur postsäkularen Zeit und zur Integration, dass man nach einer langen Irrfahrt der bundesrepublikanischen Gesellschaft nun verstanden habe, die Migranten könne man nur mit ihrer Religion integrieren, sind die Verhältnisse in Bohms Film zwischen Person, Kultur und Gesellschaft unbestimmt und inkonsistent. Inwieweit ist der Vater Yasemins ein Repräsentant und Vollstrecker eines Ehrenkodex', wenn wir beide Teile des Films zusammennehmen? Gleiches gilt für den Vetter, der sich in einer Szene bei Yasemin dafür entschuldigt, weil er jetzt mehr auf sie aufpassen müsse. Die Schwierigkeit der kulturellen Zuordnung zwischen Akteur und Gesetz zeigt sich aber am stärksten in der Figur Yasemins, die ihre Ablehnung gegenüber Ehrenkodizes immer wieder äußert und ihre Selbstbestimmung in der Aussage kulminiert, dass sie ein zufälliges Produkt zweier anatolischer Bauern sei. Dennoch ist die emotionale Bindung zwischen ihr und ihrer Familie positiv stark, nicht aber die zu irgendeiner Kultur. Bei Yasemin haben wir es mit einer Assimilation ohne Ankunft zu tun, die in viele Richtungen weist. Diese identifikative Unbestimmtheit wird jedoch in erster Linie nicht zum Problem, weil ein Ehrenkodex greift, denn dieser wird nur in Ausnahmefällen aktualisiert. Vielmehr wird die Mischung aus Assimiliertsein und Unbestimmtheit in der Person Yasemins zum Problem, weil die andere, die deutsche Seite in diesem, aber auch in vielen anderen filmischen, literarischen und auch wissenschaftlichen Publikationen der 1980er keinen Alltag und keine Heterogenität hat und somit auch keinen Ort der Ankunft. So ist Hark Bohms Film die Geschichte einer blockierten Integration, die zwischen Umarmung und Abkehr pendelt. Ein unentschiedenes Verhältnis, dessen Grundlage nicht allein die

7 Siehe zur Darstellung und Kritik des Narrativs des entleerten und entfremdeten deutschen Alltagslebens im Neuen Deutschen Film und in der Gesellschaftskritik der 1960er und 1970er Jahre: Ezli 2012b: 263-266 und Rauer 2012. 
Spannung zwischen ,guter“ Mensch und Ehrenkodex ist, sondern vielmehr auch das Misstrauen gegenüber einer Gesellschaft, die nicht empfangsfähig ist.

\section{INVERSION DER INTEGRATION ODER DER ISLAM ALS ORT DER ANKUNFT}

Zwanzig Jahre später, im Film Meine verrückte türkische Hochzeit, geht es auch um eine deutsch-türkische Liebesgeschichte, die jedoch ganz andere Verhältnisse zwischen Akteur, Gesellschaft und Kultur setzt - Verhältnisse, die den Ausführungen von Habermas und Schäuble auf den ersten Blick entsprechen, jedoch - und das wird die Analyse zeigen - genauso fragil sind wie in Bohms Film.

Meine verrückte türkische Hochzeit war ebenfalls ein äußerst erfolgreicher Film. In den Jahren 2005 und 2006 von Pro 7 produziert, erstmals 2006 ausgestrahlt, erhält der Film in den Jahren 2006 und 2007 den Grimme Preis, den Preis der deutschen Akademie der darstellenden Künste, wird Gewinner des 3sat-Zuschauerpreises und erhält je den Deutschen und Bayerischen Fernsehpreis. In den Preisbegründungen wird besonders hervorgehoben, dass es dieser Multi-Kulti-Komödie gelungen sei, alle möglichen Klischees über türkische Familien zu versammeln und gekonnt mit ihnen umzugehen. Und es sei zudem besonders über die Figur des Vaters im Film gelungen, das Ernste der Thematik ins überspitzt Komische zu überführen. Ein gelungener Umgang mit Klischees benötigt jedoch als Grundlage den Referenzrahmen einer komplexen und heterogenen sozialen Ordnung, sollen vorgeprägte Schemata in Frage gestellt werden. Der Referenzrahmen in diesem Film baut jedoch auf einer anderen Grundlage auf. Doch bevor wir zu dieser kommen, kurz die Synopse des Films:

Der Protagonist Götz Schinkel (Florian David Fitz) ist mit seinem Freund Horst (Charly Hübner) zusammen Besitzer eines Plattenladens in Kreuzberg. Sie sind die letzten Deutschen in diesem Stadtteil in Berlin und der Erzähler im Film stellt Götz zu Anfang als jemanden vor, der zu gut ist für diese Welt - im Besonderen für den von Türken dominierten Stadtteil. Dieses ,zu gut für die Welt' wird in den ersten Szenen dadurch untermauert, dass Götz zweimal vom selben Türken eine Kopfnuss bekommt: einmal als er einem türkischen Jungen helfen will und das zweite Mal in der dar- 
auffolgenden Sequenz als sich eine Menschentraube von Leuten um Götz Plattenladen bildet, weil das Fenster des Geschäfts durch eine Mecca-ColaDose zerschlagen wurde und er schlichtend eingreifen will. Schlichtend deshalb, weil Götz' Freund und Geschäftspartner Horst nach dem Zerschlagen der Fensterscheibe die Türken als islamistische Terroristen beschimpft und die Situation eskaliert. Götz liegt nach der zweiten Kopfnuss aus der Nase blutend am Boden und die schöne Türkin Aylin (Mandala Tayde), die aus der Menschentraube hervortritt, hilft ihm, in die er sich dann sofort verliebt. Aber auch die anderen Türken helfen ,als liberale Muslime“ Horst und Götz kurz darauf und richten den beiden ein neues Fenster ein. Götz zieht sich derweil in seinen Plattenladen zurück und denkt an Aylin.

Das interkulturelle Gefüge, das sich in den ersten Sequenzen aus Stadtteil, Nachbarschaft, Religion, Konflikt und Liebe narrativ nah und dicht aneinanderreiht, ist die Grundlage von Meine verrückte türkische Hochzeit, die eine invertierte Integrationsgeschichte mit einigen prekären Etappen erzählt. Denn im weiteren Verlauf des Films wird Götz versuchen, Aylins Herz und die Zustimmung ihrer Eltern, besonders ihres Vaters Süleyman, (Hilmi Sözer) zu gewinnen, die dafür aber von ihm verlangen, Moslem zu werden. Erschwerend kommt hinzu, dass Aylin mit ihrem Cousin Tarkan (Gandi Mukli) seit einem Jahr verlobt ist. Dieser ist Moslem und hat als Arzt einen finanziell sicheren und gesellschaftlich angesehenen Beruf. Dennoch verliebt sich Aylin in Götz und dieser wird den Ramadan fasten, sich beschneiden lassen und das islamische Einheitsbekenntnis vor Zeugen aussprechen. In diesem ganzen Prozess entwickelt sich im Besonderen Götz, der von einem zu guten zu einem fordernden und teilweise gewalttätigen Deutschen wird. Dieser dramaturgische Verlauf wird zwar immer wieder durch die Form der Komödie konterkariert und auch die Charaktere wirken überzeichnet. Was aber jedoch nicht zur Disposition der Umkehrung in diesem Film steht, ist die Rolle und Funktion des Islam und seines Regelwerks. Seine stabile und unverrückbare Position in diesem Film wird besonders dann deutlich als Götz Süleyman vor versammelter Familie fragt, warum er denn dagegen sei, dass er und Aylin zusammenkommen.

„Süleyman, sag doch einfach, ist es, weil ich kein Moslem bin oder weil du mich nicht magst?“ Süleymann erwidert überzeugend: „Nein, keine private Problem, aber so sind Spielregeln.“ Kurz zuvor machte Süleymans Sohn Yusuf (Ercan Özçelik) in derselben Szene klar, dass zwar ein Moslem eine Ungläubige heiraten könne, aber nicht eine Muslima einen Ungläubi- 
gen, denn sonst würde der Islam aussterben, da die Kinder in der Religion der Väter erzogen werden. Diese Szene wird, wie alle anderen Szenen im Film, zwar komödiantisch aufgelöst, aber an diese immense Sichtbarkeit des Islam schließt sich eine Leitunterscheidung an, die die eigentliche Grundlage der Integration von Götz in die türkische Familie gewährleistet. An die Aussage von Götz, dass er nun Moslem werde, worüber Süleyman nicht erfreut ist und mit der Gegenaussage quittiert, „,bevor Du Moslem wirst, wird Bin Laden Weihnachtsmann“, schließt sich ein Zwiegespräch zwischen Melek und Süleyman in der Küche an. „Mach es diplomatisch“, sagt sie ihm. „Ein Deutscher schafft nie den Ramadan.“

Doch Götz erfüllt alle Auflagen, ist integrationswillig und zeigt sich am Ende als starker und ernst zu nehmender Mann. Dass die Unterscheidung stark/schwach eine Leitkategorie der Integration in diesem Film darstellt, zeigt sich auch besonders am Ende von Meine verrückte türkische Hochzeit. Als die Verlobung zwischen Götz und Aylin im Haus von Götz Mutter (Katrin $\mathrm{Saß}$ ) eskaliert, weil sie dagegen ist, dass die Kinder ihres Sohnes im islamischen Glauben erzogen werden - bis zu dieser Szene wusste sie nichts von der Konversion ihres Sohnes - kippt der Film in einen Kulturkampf. Für sie ist der Islam eine Religion, ,die Millionen von Frauen durch Zwangsehen unterdrückt". ${ }^{8}$ Gegenseitige Beleidigungen führen in dieser Sequenz dazu, dass Aylins Familie das Haus verlässt. Götz beschwichtigt darauf seine Mutter und beide fahren der türkischen Familie nach und es folgt die dramatischste Sequenz des Films, die nicht irgendwo im Privaten, sondern mitten auf der Straße in Kreuzberg stattfindet.

Vor der Wohnung Süleymans erreicht Götz die türkische Familie und will über das Geschehene reden. Yusuf reagiert mit einem Faustschlag in Götz' Magengrube und der Aussage, dass er an allem Schuld sei, und stößt ihn auf ein parkendes Auto. Der türkische Mann, der zu Anfang des Films Götz eine Kopfnuss verpasst hatte, eilt herbei, da es sich bei dem Auto um seines handelt und verlangt von Götz Geld für den entstandenen Sachschaden. Götz zückt aufgebracht seinen Geldbeutel, wirft das Geld auf den Boden und beginnt seine Wutrede, dass sie nun sein Geld haben, seine Religi-

8 Helena stellt in diesem Film eine Alt-68er Feministin dar, die für verstaubte Ideale des individuellen und freien Glücks steht. Sie hat im Film keinen Partner und es erschließt sich auch nicht, ob sie jemals verheiratet war. Götz' biologischer Vater wird mit keinem Satz erwähnt. 
on, seine Vorhaut und was sie denn noch sonst alles wollten. Diese Straßensequenz wird immer intensiver und beleidigender für beide Seiten. Götz wird vorgeworfen, dass er „Schiss“ habe und kein richtiger Mann sei und jetzt alles „hinschmeißen“ wolle. Götz reagiert darauf, dass sie Schiss hätten und sich hinter ihrer „,verfickten Ehre“ und Religion verstecken würden und sie „Scheißtürken“ und „Rassisten“ seien. Die Szene endet damit, dass Götz Aylin sagt, dass sie in Wirklichkeit Angst habe, er sie aber trotzdem liebe. Sie wendet sich mit einem ,vergiss es“ ab und zieht sich mit ihrer Familie zurück und Tarkan ist nun auf Anraten ihrer Familie wieder der Auserwählte. Sie fügt sich dem mit Widerwillen.

Götz, an einem mentalen Endpunkt angekommen, rächt sich an Tarkan, der ihm zuvor sein Auto zerschlagen hatte, als es noch so aussah, dass Götz und Aylin heiraten würden. Er holt sich bei Süleyman in dessen Trödelgeschäft einen Besen und zerschlägt mit diesem mitten auf der Straße das Auto von Tarkan. Er gibt darauf den Besen bei Süleyman wieder ab und Süleyman lädt ihn dann auf ein, zwei Rakis in seinem Geschäft ein und führt mit ihm zum ersten Mal ein Gespräch auf Augenhöhe: er rät ihm, Aylin wieder zurückzuholen. Götz folgt seinem Rat und durch sein Eingreifen in die Trauung zwischen Aylin und Tarkan werden Götz und Aylin durch den Imam in einer sehr tumultartigen Straßenszene - Aylins und Tarkans Familie kämpfen auf der Straße miteinander - in Götz' Auto vermählt. Der Film endet abends mit der Hochzeitsfeier von Aylin und Götz vor seinem Plattenladen, von wo aus der Film mit dem Zerschlagen der Fensterscheibe seinen Anfang genommen hatte.

Vom Sender Pro 7, der diesen Film produzierte, wurde er 2006 als eine Romantic Comedy beworben. Und man muss diesem Film sicher attestieren, dass es sich insgesamt um eine Multi-Kulti-Komödie handelt - jedoch nicht um eine Feelgood-Comedy wie My Big Fat Greek Wedding (2002), mit dem Meine verrückte türkische Hochzeit verglichen wurde. Vielmehr finden wir in diesem Film auch die politische Disposition der deutschen Migrationsgesellschaft zu Beginn des 21. Jahrhunderts vor. In ihr sind, wie zu Anfang mit Habermas' und Schäubles politischen Diktionen kurz skizziert, die Türken und ihre Kindeskinder, die vor fünfzig Jahren aus der Türkei nach Deutschland emigrierten, nun in der Mitte der Gesellschaft, nicht als Südländer, nicht als Orientalen, nicht als Kanaken und auch nicht als Europäer, sondern als Muslime angekommen. Und so sind auch die 
deutsch-türkischen Akteure in Meine türkische Hochzeit vom Islam nicht zu trennen. Als sich Helena und Aylin das erste Mal bei einer Buchpräsentation von Helena begegnen, wird Aylin von einer Freundin Helenas, die über Zwangsehen und Ehrenmorde ein Buch verfasst, gefragt, warum die türkischen Mädchen sich das alles gefallen lassen würden. Aylin entgegnet in der komödiantischen Diktion des Films: „Ach, das ist ganz normal. Wir türkischen Mädchen sind ein bisschen doof, weil wir in den Koranschulen nur kochen, putzen und beten lernen, und bei diesen ganzen Schlägen auf den Kopf. Aber ich habe mir heute zum ersten Mal eine Monatskarte gekauft und Götz bringt mir gerade das S-Bahn fahren bei. Extrem aufregend.“ (Holtz 2007) Götz, von der Reaktion seiner Freundin angetan, ergänzt, dass Aylin Jura studiere. Helena versucht die peinliche Situation zu lockern und schenkt Aylin ihr Buch „Happy Single“ mit einer Widmung. Aylin reagiert auf diesen Buchtitel weiter selbstbewusst, jetzt ohne komödiantischen Dreh, dass sie nicht ein happy single, dafür aber ein „Happy Muslim"sei.

Ebenso wie Yasemin in Hark Bohms Film trägt Aylin kein Kopftuch, ist modern gekleidet und ebenso auf kognitiver, struktureller und sozialer Ebene als assimiliert zu bezeichnen. Jedoch kommt am Ende dieser Kette bei Aylin weder eine Verschmelzung mit der Mehrheitsgesellschaft noch ein happy single heraus, sondern vielmehr eine Identifikation, die die kulturelle Kontaktzone nicht in Individuen, sondern in Kollektive einteilt. Diese Form der kollektiven Identifikation, die die türkische und auch deutsche Seite in Meine verrückte türkische Hochzeit betrifft, zeigt sich besonders in den beschriebenen Filmsequenzen, die diese Multi-Kulti-Komödie ins Dramatische und Beleidigende kippen lassen. Wenn der Islam oder die Freiheit verbal verletzt werden, fühlen sich die Personen verletzt. Kein Akteur in diesem Film kann auf eine Beleidigung mit einer kulturell heterogenen Struktur seines Alltags und seiner Person reagieren, weil diese nicht gezeigt und auch nicht dokumentiert wird. Zu sehr dominieren hier Abstrakta wie Islam, Moslem, Türke, Deutscher mit ihren materiellen Repräsentationen wie Moschee, Kopftuch und Koran. Dagegen erschöpft sich das Potential des Komischen in diesem Film lediglich darin, diese Abstrakta umzukehren und nicht in Richtung einer heterogenen Sozialordnung zu weisen. Im Unterschied zu Bohms Film bricht so auch kein kulturelles Gesetz in diesen Film ein und irritiert eine vorhandene soziale Fluidität. Die Kulturen sind 
von Anfang an prozessunabhängig und monolithisch präsent und das Verhältnis von Personen und Regeln ist ein enges. Auch wenn diese immer wieder karikiert werden, erzeugt die narrative Dichte und Folge von Komik, Gewalt, Liebe und Beleidigung ein multikulturelles Unbehagen, das sich in den dramatischen Sequenzen zwar entlädt, aber nicht aufgefangen werden kann. So gibt es hier nicht ein Problem der Ankunft, sondern vielmehr eines der Aufnahme, die ein sozial fluides und zugleich stabiles Verhältnis zwischen Akteur und Religion ermöglichen könnte - ein Verhältnis, das in folgender Haltung kulminieren könnte, nicht alles, was über den Islam oder den Westen gesagt wird, löst Identifikation und Betroffenheit aus. Da diese Technik der Entdramatisierung und Verhandlung fehlt, ist auch das Gefühl am Ende des Films bei der Hochzeit nicht eines der Erleichterung. Vielmehr schleicht sich die Frage ein, wie lange diese Ehe oder Integrationsallianz halten wird.

\section{FAZIT}

Beide Filme, Yasemin und Meine verrückte türkische Hochzeit, waren äußerst erfolgreiche und breitenwirksame Filme, obwohl sie im Unterschied beispielsweise $\mathrm{zu}$ beliebten Hollywoodproduktionen aus jeweils unterschiedlichen Gründen lose Enden haben und keine stabilen Ankunftsnarrative aufbauen (Vgl. Koschorke 2012: 137-142). Die Integration in Bohms Yasemin ist blockiert, weil es keinen Ort der Ankunft gibt. Diese blockierte Integration findet sich auch in den Debatten und politischen Entscheidungen zwischen Ende der 1970er bis Ende der 1980er Jahre, deren Grundlage die politische Diktion „Deutschland ist kein Einwanderungsland“ war (siehe Herbert 2001: 249-262), obwohl diese der sozialen Wirklichkeit widersprach. Die Historiker Ulrich Herbert und Karin Hunn verweisen in diesem Zusammenhang beispielsweise auf die Debatte, die 1979 der österreichische Verhaltensforscher Irenäus Eibl-Eibesfeldt mit seiner Aussage lostrat, dass Türken auch nur Menschen seien, aber Menschen mit einer anderen Kultur - und deshalb ihre Integration problematisch sei (Vgl. Hunn 2005: 495). Hark Bohm ist sicher ideologisch von Eibl-Eibelsfeldt weit entfernt und wie auch schon zu Anfang festgehalten, ging es Bohm bei seiner Geschichte von Yasemin um eine archaische, die nicht die Gesellschaft und im Speziellen nicht die Kultur im Visier hatte, sondern eine zu bildende Ge- 
meinschaft. Dennoch folgt sein Film der narrativen Folge der Aussage Eibesfeldts: zuerst ,guter“ Mensch, dann die verstörende in der Türkei angesiedelte Kultur. Auf die soziale Fluidität folgt der Einbruch eines Regelwerks, der den deutschen Beobachter irritiert zurücklässt. Eine Irritation, die weit mehr mit dem Problem der eigenen Selbstverortung zu tun hat, als mit der Verortung der türkischen Ehre (Vgl. Ezli 2009: 213 f., 217-220).

In Meine verrückte türkische Hochzeit gibt es keine so divergierenden Distanz- und Nahverhältnisse zwischen Mensch und Kultur. Vielmehr bilden sie dort von Anfang an eine Einheit. Es dominiert hier die Quintessenz der deutschen Integrationspolitik seit 2005, dass man die Türken nur mitsamt ihrer Religion integrieren könne und sie auch als solche nun angekommen seien. Bei dieser identitätspolitischen und symbolischen Ankunft handelt es sich um eine andere Form der Kulturalisierung als in den 1980er Jahren; um eine, mit der nicht mehr die Geschichte des gebrochenen ,guten“ Ausländers erzählt wird, sondern Akteure dargestellt werden, die ohne einen engen Bezug zu Kultur als Regelwerk, Gefahr oder als ökonomisches Potential nicht mehr gedacht werden können.

Beide Filme, die unterschiedliche Geschichten der Integration erzählen, zeigen mit ihren gesellschaftspolitischen Rahmungen, dass Integration nicht nur eine Frage der Perspektive zwischen Modellen der Assimilation oder der Diversität ist, sondern auch eine zentrale Frage der Erzählung. Denn Erzählungen ,,verarbeiten und gestalten Komplexität durch Dynamisierung, durch Auflösung von Zustand in Prozess“ (Koschorke 2012: 21). Im Unterschied zu Assimilations- oder Integrationskonzepten stehen hier weder Istoder Sollzustände von Gesellschaften als Ursache oder als Ziel im Vordergrund, sondern vielmehr Prozesse im Fokus als Abfolgen sozialer Handlungen. „So betrachtet, stellt das Erzählen ein in hohem Maße unspezifisches Medium dar. [...] Es bildet eher ein Sammelbecken von Redeweisen [...]; seine kulturelle Leistung liegt weniger im Trennen als im Verbinden“ (Koschorke 2012: 38). Doch trotz dieser Offenheit muss das Erzählen, will es sich wie jede sprachliche Information mitteilen, ,stillschweigend auf einen Fundus an sensorischen und praktischen Erfahrungen Bezug nehmen; es muss in vieler Hinsicht ein unartikuliertes Vorwissen aufrufen können“, um in der Zone zwischen Alltag, Kunst und Politik Wahrscheinlichkeiten $\mathrm{zu}$ artikulieren. So steht dieser, ,informellen und in gewisser Weise einladenden Offenheit [der Erzählung] die Gravitationskraft bestimmender kulturprägender Narrative entgegen“ (Koschorke 2012: 38). 
In den hier untersuchten Integrationserzählungen bündeln sich in einem Fall die Sichtweisen der 1980er Jahre. Der Einbruch des Kulturellen zerschlägt die narrativen Konventionen erwarteter Abfolgen. In der zweiten Erzählung, Meine verrückte türkische Hochzeit, ist das soziologische Konzept der 1990er und 2000er Jahre nahezu gespiegelt. Dieses Konzept fordert, dass sich auch die aufnehmende Kultur anzupassen und zu wandeln, d.h. - zu integrieren habe. Götz unternimmt als Figur diese neue Integrationsrichtung tatsächlich. Jedoch ist der Ausgang in Meine verrückte türkische Hochzeit ebenso instabil wie bei Bohms Film Yasemin. So sind in beiden Filmen die Übergänge und Abfolgen von Ist- zu Sollzuständen problematisch und sie verlangen mit ihren damit verbundenen losen Enden nach anderen und stabileren Narrativen der Integration, die den prozessabhängigen heterogenen Bindungen zwischen Akteur, Kultur und Gesellschaft entsprechen und sie aufnehmen können.

\section{LITERATUR}

Alabay, Başar. Kulturelle Aspekte der Sozialisation. Junge türkische Männer in der Bundesrepublik Deutschland. Wiesbaden: VS Verlag, 2012.

Bhabha, Homi K. The Location of Culture. London: Routledge 1994.

Bohm, Hark / Weinert, Rita / Roth, Wilhelm. „Plädoyer für das Erzählkino. Gespräch mit Hark Bohm." In: epd Film, April 1988, S. 2-5.

Ehlers, Ulrich. „Yasemin. Ein Film für Zwölfjährige.“ In: Bundesverband Jugend und Film (Hg.). Teenie-Kino. Filmarbeit zwischen Kindheit und Jugend. Frankfurt a. M.: Peter Lang 1993.

Esser, Hartmut. Aspekte der Wanderungssoziologie. Assimilation und Integration von Wanderern, ethnischen Gruppen und Minderheiten. Eine handlungstheoretische Analyse. Darmstadt, Neuwied: Luchterhand, 1980.

Ezli, Özkan. Grenzen der Kultur. Autobiographien und Reisebeschreibungen zwischen Orient und Okzident. Konstanz: Konstanz University Press 2012a.

Ezli, Özkan. ,' Auf Empfang eingestellt... ‘“. Autokommunikation als kulturelle Dynamik in Rainer Werner Fassbinders Angst essen Seele auf (1973)“. In: Frank, Susi/Ruhe, Cornelia/Schmitz, Alexander (Hg.). Exp- 
losion und Peripherie: Jurij Lotmans Semiotik der kulturellen Dynamik revisited. Bielefeld: transcript 2012b, S. 247-267.

Ezli, Özkan. „Von der interkulturellen zur kulturellen Kompetenz: Fatih Akıns globalisiertes Kino“. In: ders./Kimmich/Werberger. Wider den Kulturenzwang. Migration, Kulturalisierung und Weltliteratur. Bielefeld: transcript, S. 207-230.

Faist, Thomas. „Jenseits von Nation und Postnation. Eine neue Perspektive für die Integrationsforschung“. In: Ders. (Hg.). Transstaatliche Räume. Politik, Wirtschaft und Kultur in und zwischen Deutschland und der Türkei. Bielefeld: transcript 2000, S. 339-394.

Geis, Matthias. „Perspektiven durch Kontraste in einem Klassiker des Fremderlebens. Yasemin von Hark Bohm.“ In: Praxis Deutsch, 29 (2002) 175, S. 35-43.

Gordon, Milton M. Assimilation in American Life. The Role of Race, Religion and National Origin. New York: Oxford University Press, 1964.

Han, Petrus. Soziologie der Migration. Stuttgart: Lucius \& Lucius, 2009.

Herbert, Ulrich. Geschichte der Ausländerpolitik in Deutschland. Saisonarbeiter, Zwangsarbeiter, Gastarbeiter, Flüchtlinge. München: C. H.Beck 2001.

Hunn, Karin. „Nächstes Jahr kehren wir zurück“. Die Geschichte der türkischen „Gastarbeiter“ in der Bundesrepublik. Göttingen: Wallstein Verlag 2005.

Koschorke, Albrecht. Wahrheit und Erfindung. Grundzüge einer allgemeinen Erzähltheorie. Frankfurt a. M.: Fischer 2012.

Ören, Aras. Was will Niyazi in der Naunynstraße?. Berlin: Rotbuch 1973.

Park, Robert Ezra. „Human Migration and the Marginal Man.“ In: AJS 33,1928, 881-893.

Rauer, Valentin. Die öffentliche Dimension der Integration. Migrationspolitische Diskurse türkischer Dachverbände in Deutschland. Bielefeld: transcript, 2008.

Rauer, Valentin. „Imagination des Minoritären. Terroristen in Fassbinders ,Die Dritte Generation““. In: Colin, Nicole et al. (Hg.): Prekäre Obsession. Minoritäten im Werk von Rainer Werner Fassbinder. Bielefeld: transcript 2012, S. 353-368.

Schäuble, Wolfgang: Rede zur Eröffnung der 4. Plenarsitzung der Deutschen Islam Konferenz (DIK) am 25. Juni 2009. Auf: http//deutsche-islam-konferenz.de (Stand 20.01.2012). 
Schiffauer. Werner. Die Gewalt der Ehre. Erklärungen zu einem türkischdeutschen Sexualkonflikt. Frankfurt a. M.: Suhrkamp 1983.

Tezcan, Levent. Das muslimische Subjekt. Verfangen im Dialog der Deutschen Islam Konferenz. Konstanz: Konstanz University Press 2012.

Visarius, Karsten. „Ehrenrettung um jeden Preis. Zu ,Yasemin“ von Hark Bohm“. In: Karpf, Ernst/ Kiesel, Doron/ ders. (Hg.). Getürkte Bilder. Zur Inszenierung von Fremden im Film. Marburg: Schüren 1995, S.117-124.

Yıldız, Yasemin. „Kritisch ,Kanak‘. Gesellschaftskritik, Sprache und Kultur bei Feridun Zaimoğlu“. In: Ezli, Özkan / Kimmich, Dorothee/ Werberger, Annette (Hg.). Wider den Kulturenzwang. Migration, Kulturalisierung und Weltliteratur. Bielefeld: transcript 2009, S. 187-206.

\section{DVD}

YASEMIN (Deutschland 1988, R: Hark Bohm)

MEINE VERR ÜCKTE TÜRKISCHE HOCHZEIT (Deutschland 2007,

R: Stefan Holtz)

MY BIG FAT GREEK WEDDING (Kanada 2002; R: Joel Zwick) 